\section{Measurement of fornix depth and area: a novel method of determining the severity of fornix shortening}

T Kawakita ${ }^{1,2}$, M Kawashima', D Murat, ${ }^{1,2}$, K Tsubota ${ }^{1,2}$ and J Shimazaki ${ }^{1,2}$
'Department of Ophthalmology, Tokyo Dental College Ichikawa General Hospital, Chiba, Japan

${ }^{2}$ Department of Ophthalmology, Keio University, Tokyo, Japan

Correspondence:

T Kawakita, Department of Ophthalmology, Tokyo Dental College, Sugano 5-11-13, Ichikawa City,

Chiba,

Japan

Tel: + 8147322 0151;

Fax: + 81473226786

E-mail: kawakita@

sc.itc.keio.ac.jp

Received: 26 March 2008 Accepted in revised form: 2 June 2008

Published online: 11 July 2008

Authors contributions: TK: conception and design, analysis and interpretation, writing of the draft paper; MK: data collection, provision of patient materials; DM: analysis and interpretation, writing of the draft paper; KT: analysis and interpretation; JS: analysis and interpretation

Competing interests: None 
study. None of the patients and control subjects had a history of any other ocular or systemic disease, history of ocular surgery or systemic drug use, trauma or radiation exposure that would have caused cicatricial forniceal changes. Informed consents were obtained from all participants before measuring the fornix depth.

Examination procedures were reviewed by Ethics Board.

\section{Fornix depth measurements}

The authors devised a dull-edged stainless steel rod measuring $15 \mathrm{~cm}$ in length and $2 \mathrm{~mm}$ in diameter with a millimetric scale at both ends. The rods were sterilized before measurements and were held from the central part. The presence of a millimetric scale at both ends allowed the measurement of fornix depth in a sterile manner for both eyes. The fornix depth measurements were carried out at six points in each eye. Briefly, the vertical fornix depths were measured from the superior nasal and temporal as well as the inferior nasal and temporal conjunctival fornices at lines tangential to the nasal and temporal limbus in each eye. The horizontal fornix depths were measured from the inner and outer canthi. Measurements were performed under slit lamp observation. Before measurement, all subjects were instructed to look at a fixation light presented at a fixed angle. The rod (as shown in Figure 1a and b) was gently inserted into the conjunctival sac under topical anaesthesia with $1 \%$ oxybuprocaine hydrochloride (Benoxyl; Santen Pharmaceuticals, Osaka, Japan).

\section{Fornix area measurements}

Anterior segment slit lamp photographs were taken under $\times 5$ magnification. A ruler with a millimetric scale was placed under the lower eyelid and was included in each photo frame (Figure 1c) to help in area calculations using the NIH Image J software (Bethesda, MD, USA).
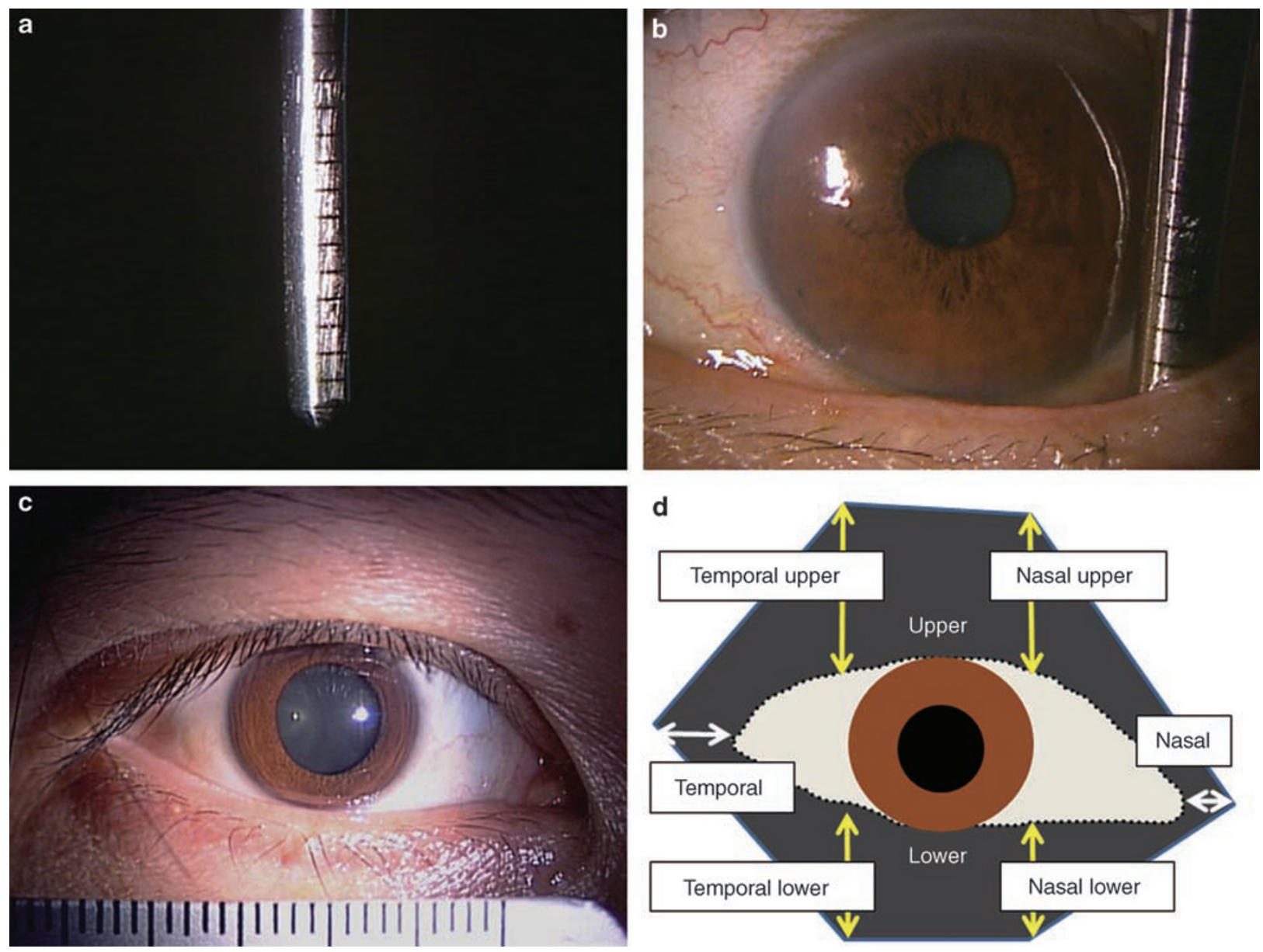

Figure 1 The 'fornicometer' instrument and measurement of the fornix depth. Note the millimetric scale at the end of the fornicometer (a). The instrument is gently inserted into fornix under topical anaesthesia (b). Anterior segment photograph of a control subject with a scale bar below the lower eyelid serving as a reference for fornix area calculations (c). Schematic diagram showing the sites of fornix depth measurements and construction of the estimated overall fornix area (d). 
a

Fornix Depth

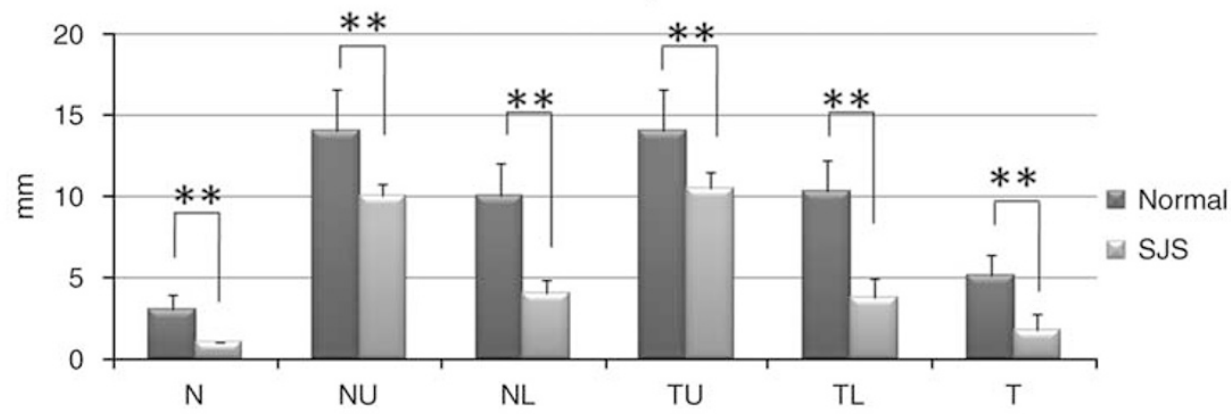

b

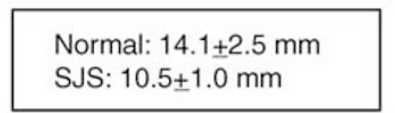

Normal: $5.2 \pm 1.2 \mathrm{~mm}$

SJS: $1.75 \pm 0.95 \mathrm{~mm}$

Normal: $10.0+2.1 \mathrm{~mm}$

SJS: $3.75 \pm 1.17 \mathrm{~mm}$

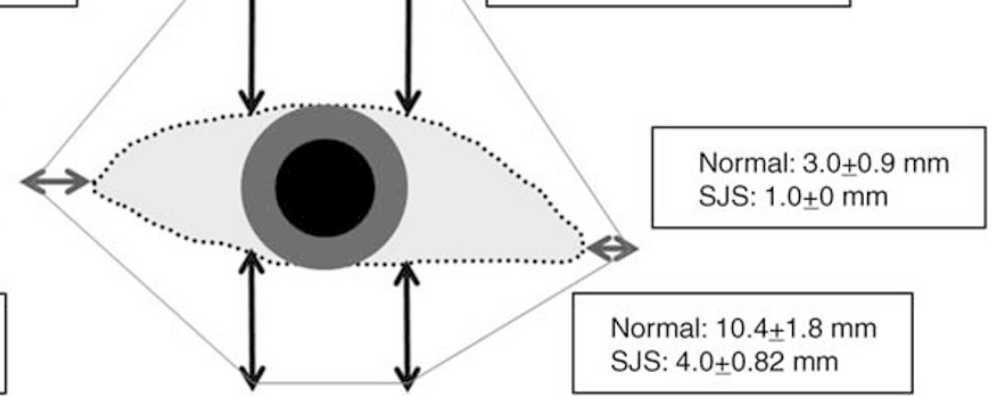

Figure 2 Comparison of fornix depths between normal subjects and patients. Note the statistically significant shortening of fornix depths at all measurement locations in the patients $(P<0.001)$. (a) Note the differences of mean fornix depths between Stevens-Johnson syndrome (SJS) patients and control subjects. Schema of fornix depth at 6 points was shown (b). N: nasal; NU: nasal upper; NL: nasal lower; TU: temporal upper; TL: temporal lower; T: temporal.

Lines showing the fornix depth measurements were drawn from the edge of upper and lower eyelids. The interpalpebral and intercanthal distances were also drawn on a schematic diagram. The tip of the lines from the six measurement sites were connected to construct an estimate for the overall forniceal area (Figure 1d). The NIH Image J software automatically displayed the number of pixels for each $1 \mathrm{~mm}^{2}$ area. The total number of pixels was then divided by the number of pixels per square millimetre. This calculation was performed separately for the upper and lower forniceal area constructs, the sum of which provided the overall forniceal area estimate. Fornix depth and area measurements were also carried out before and after ocular surface reconstruction surgery with amniotic membrane transplantation in one patient.

Before and after measurements of the fornix depths, the ocular surface in each subject was stained by $0.5 \%$ fluorescein dye instilled with a micropipette to investigate the degree of invasiveness of the measurement on the ocular surface.

\section{Statistical analysis}

Student's $t$-test was used for the comparison of fornix depth and areas between patients and control subjects.
Instat program (GraphPad Software, CA, USA) was used for statistical analysis. A $P$-value less than 0.05 was considered as statistically significant.

\section{Results}

\section{Fornix depth measurements}

The mean vertical fornix depths measured from the upper nasal and temporal as well as the lower nasal and temporal fornices in the control eyes were $14.4 \pm 2.7$, $14.9 \pm 2.6,10 \pm 2.2$, and $10.6 \pm 2.7 \mathrm{~mm}$, respectively. The mean horizontal fornix depths in the control subjects measured $3.2 \pm 0.1$ and $5.1 \pm 0.1 \mathrm{~mm}$, respectively. These values were significantly higher compared to the corresponding measurements in the patient eyes $(P<0.01)$ as shown in Figure 2.

\section{Fornix area measurements}

The average forniceal area in normal subjects was $909.6 \pm 162.2 \mathrm{~mm}^{2}$ (upper fornix area: $510.0 \pm 59.4 \mathrm{~mm}^{2}$; lower fornix area: $399.6 \pm 102.8 \mathrm{~mm}^{2}$ ) (Figure 3). These values were significantly lesser in patient eyes compared with the control subjects $(P<0.01)$. None of the subjects 
a

Fornix Area
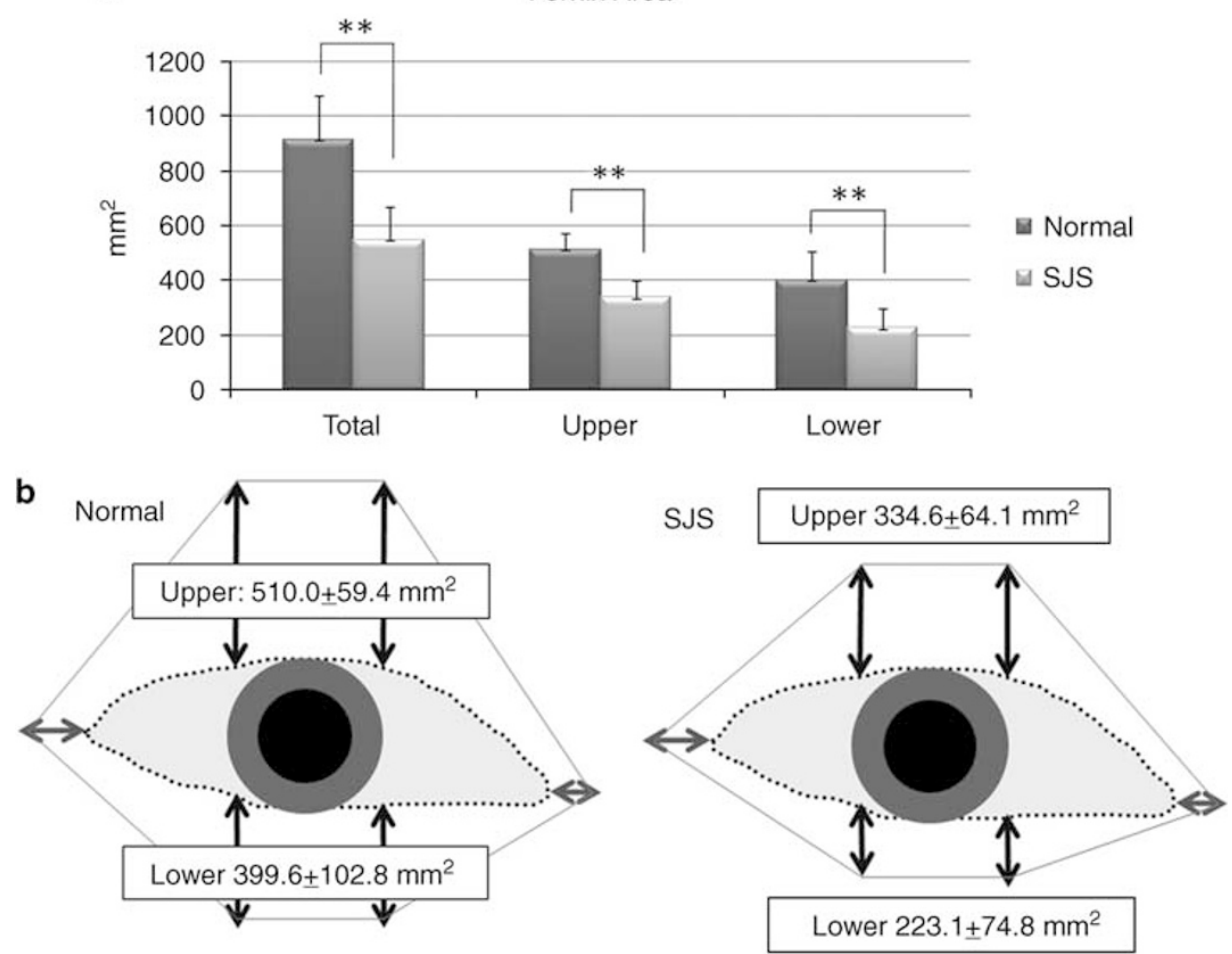

Figure 3 Comparison of fornix areas between normal subjects and patients. Note the significant narrowing of the overall fornix area estimates in the patients $(P<0.001)$ (a). Note the differences of fornix area estimates between Stevens-Johnson syndrome (SJS) patients and control subjects (b).

complained of discomfort or other symptoms, during fornix depth measurements.

\section{Fornix depth/area measurements before and after ocular surface reconstruction surgery}

Temporal forniceal depth and overall fornix area showed improvement after fornix reconstruction surgery in a patient with ocular surface cicatrisation as shown in Figure 4 .

\section{Complications}

No post-measurement conjunctival fluorescein staining or conjunctival injection was observed in any of the subjects. No subject complained of pain or foreign body sensation during or after the measurements.

\section{Discussion}

Grading of conjunctival injection, the extent of loss of palisades of Vogt, and quadrants of corneal vessel invasion have been reported as objective measures in determining the severity of cicatrizing conjunctival ocular surface diseases. Forniceal shortening has been described as one of the major complications of cicatrizing conjunctivitis, for which not a single method of quantification has been reported in the literature. We report, for the first time, a simple method for the assessment of the forniceal depth and area in patients with Stevens-Johnson syndrome comparing the results with those of healthy control subjects. The mean forniceal depth values were significantly shallower in patients compared to the control subjects in this study. Likewise, the overall fornix area was significantly smaller in patient eyes when compared with the control subjects. Our findings suggest that assessment of fornix depth and area may serve as useful quantitative parameters to describe the severity of cicatrizing ocular surface diseases. Although further studies on a larger number of patients are essential, the improvement of the same parameters after ocular surface reconstruction surgery with amniotic membrane transplantation suggests the applicability of these parameters in the evaluation of treatment success and/or prognosis. Long-term follow-up studies comparing the extent of improvement in these parameters with different reconstruction procedures may contribute to our understanding about the selection of the best surgical procedure for the cicatrizing ocular surface diseases in the future. Provision of age- and gender-specific fornix depth and area standards in 


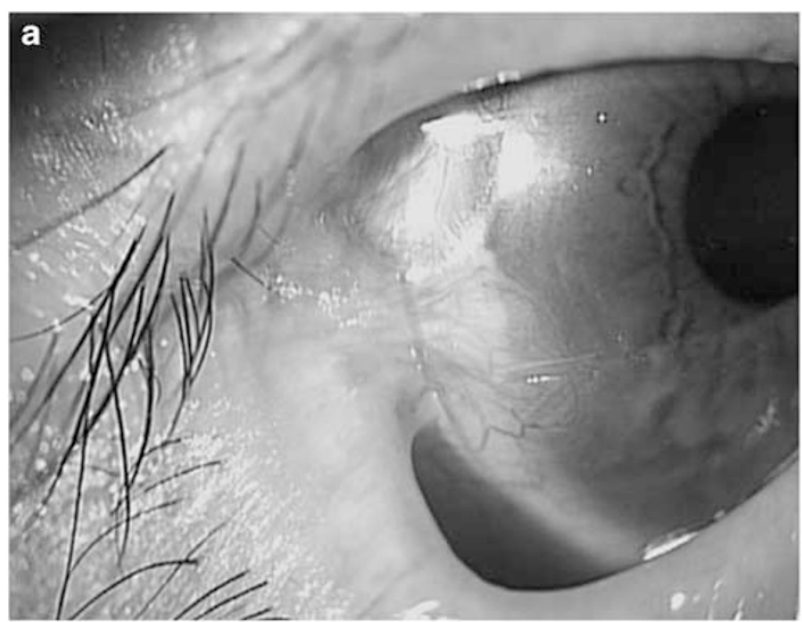

c Temporal depth before and after surgery

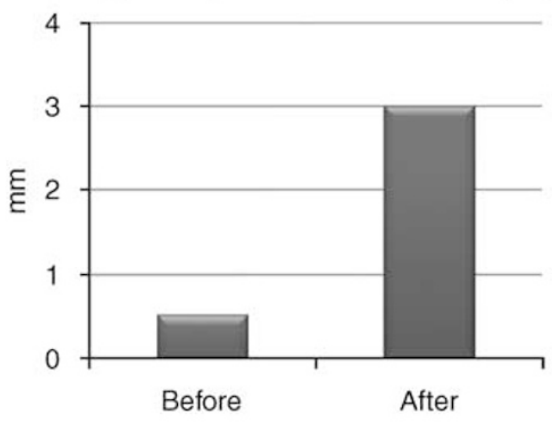

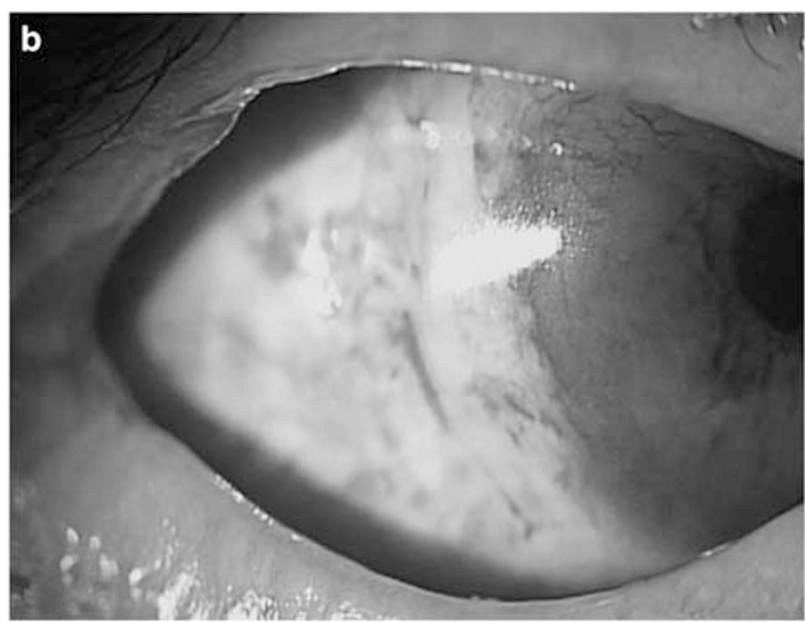

d

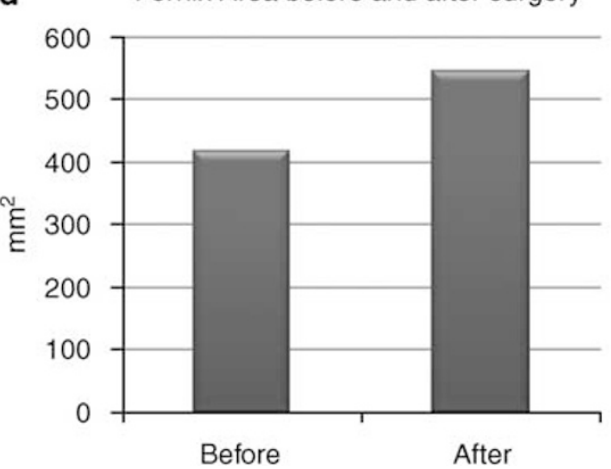

Figure 4 Evaluation of fornix depth and area before and after fornix reconstruction surgery with amniotic membrane transplantation in a patient with ocular surface cicatrization. Anterior segment photograph before fornix reconstruction (a), anterior segment photograph after fornix reconstruction (b). Note the improvement in the temporal fornix depth after surgery (c). Note also the improvement in forniceal area after surgery $(\mathrm{d})$.

healthy subjects remains the goal of future studies. The optimum number of measurement sites reflecting the severity of forniceal shortening with more precision should also be determined in further prospective controlled trials. Reproducibility of the measurements among different examiners and on different days of measurement need to be established as well. The novel methodology described by us seems to be safe and noninvasive as none of the subjects complained of foreign body sensation and/or pain following the measurements. No fluorescein staining was observed after the measurements with this method. However, this instrument cannot be used to detect the fornix obliteration due to other means such as conjunctivochalasis.

In summary, we described a new method and two novel parameters, namely, fornix depth and area for quantification of forniceal shortening in this study. These parameters may be useful in determining the severity of cicatricial ocular surface diseases and in evaluating the outcome/prognosis of various ocular surface reconstruction procedures.

\section{Acknowledgements}

We do not have any proprietary interest in any of the products mentioned in the paper. The fornicometer device is a prototype that is not commercially available.

\section{References}

1 Honavar SG, Bansal AK, Sangwan VS, Rao GN. Amniotic membrane transplantation for ocular surface reconstruction in Stevens-Johnson syndrome. Ophthalmology 2000; 107: 975-979.

2 Holsclaw DS. Ocular cicatricial pemphigoid. Int Ophthalmol Clin Fall 1998; 38: 89-106.

3 Sanfilippo P, Lee G. Ocular cicatricial pemphigoid. Clin Exp Optom 2003; 86: 47-50.

4 Solomon A, Espana EM, Tseng SC. Amniotic membrane transplantation for reconstruction of the conjunctival fornices. Ophthalmology 2003; 110: 93-100.

5 Tseng SC, Di Pascuale MA, Liu DT, Gao YY, Baradaran-Rafii A. Intraoperative mitomycin $C$ and amniotic membrane transplantation for fornix reconstruction in severe cicatricial ocular surface diseases. Ophthalmology 2005; 112: 896-903. 